\title{
Superluminescent laser-integrated nanocarbonized matrix pumping the neodymium lasers YAG:Nd
}

\author{
M.S. Onachenko \\ Institute of Microdevices, NAS of Ukraine \\ 3, Pivnichno-Syretska str., 04136 Kyiv, Ukraine. \\ Kyiv National Polytechnic University "KPI" \\ 37, prospect Peremohy, 03056 Kyiv, Ukraine \\ E-mail: maratonachenko@gmail.com
}

\begin{abstract}
Forming of surface nanocarbonized structures on substrate, it is possible to reduce the series resistance of nanoregions contacts in heterostructures. It was achieved by increasing the efficiency and output power in nanocarbonized structures by 2 times with decreasing the heat release on successive contact resistance. We obtained improved parameters of nanocarbonized matrices for pumping after finding the possibility to create a new generation of neodymium lasers with semiconductor pumping without forced cooling. The obtained III-nitride AlGaInN/Si heterostructures instead of the AlGaAs/GaAs ones allow to more accurately select and grow the better chemical composition for the emitting region, the emission spectrum of which is closer to the radiation of a neodymium laser.
\end{abstract}

Keywords: nanocarbonized structure, heterostructure, matrix for pumping, neodymium laser, YAG:Nd.

Manuscript received 19.03.15; revised version received 14.07.15; accepted for publication 03.09.15; published online 30.09.15.

\section{Introduction}

Significant progress in the development of hybridmonolithic technology of LED chips with higher power was shown in recent years. It allows to create an energyefficient $[1,2]$ integrated pumping systems for solidstate lasers, in particular, high-power neodymium lasers $(\lambda=1.06 \mu \mathrm{m})$ that are widely used in processing the construction materials. The system for LED pumping the neodymium lasers are usually created using GaAs/AlGaAs heterostructures, and it requires additional cooling with Peltier thermoelectric cooler at high powers.

Due to the technological integration of elements based on $A^{3} B^{5}$ materials proposed in 1972, significant increase in the power and quantum yield of lightemitting diodes on $\mathrm{Si}$ substrates was achieved in the last decade [4]. However, the methods that was used to reach a significant increase in the quantum yield of III-Nitride LEDs on silicon substrates (ten percent) led to such a great amount of losses that the overall efficiency of the device remains low or requires forced cooling with Peltier elements [2].

In this work, we investigated pumping the neodymium lasers infrared InN with LED and LD nanostructures of gallium, indium and aluminium nitride, grown using MOCVD selective epitaxy from organometallic compounds and ammonia on silicon substrates [2].

Small composition $\mathrm{InN}$ in $\mathrm{AlGaN}$ solid state solution has been investigated and used in LED's and LD-structures. The energy gap $E_{g}$ of InN was determined in recent years. Early absorption studies of sputtered thin films found $E_{g}$ within the 1.7 to $2.2 \mathrm{eV}$ range $[3,4]$. These polycrystalline or nanocrystalline films often had high electron densities and low-temperature gap of 
approximately $1.99 \mathrm{eV}$, which represented a dramatic reevaluation. Those results were confirmed by different groups [5]. Luminescence data for InGaN and InAlN alloys also supported the narrow-gap nature of the endpoint InN. Theories similarly revised the gap down, to $0.7 \ldots 0.9 \mathrm{eV}$, and examined why it is anomalously narrow in the context of trends for other semiconductor materials (violation of the common-cation rule).

The previous observations of the wide gap width were tentatively attributed to a large Moss-Burstein shift, InN- $\mathrm{In}_{2} \mathrm{O}_{3}$ alloying due to significant oxygen incorporation into the sputtered samples, quantum confinement effects in nanoscale grains, and deviations from stoichiometry (excess nitrogen incorporation). While the first possibility can explain the results for highly-doped samples, Butcher and Tansley pointed out that it fails to account for reports of low-doped samples with wide gaps. $\mathrm{InN}-\mathrm{In}_{2} \mathrm{O}_{3}$ alloying effects are not expected to be great enough, and in some cases there is no hard evidence for such alloys forming (although oxygen incorporation may occur). Confinement effects cannot be ruled out in some cases, but there is no indication for gap variations with grain size. It is also unclear how excess $\mathrm{N}$ would impact the gap. Compounding the confusion are recent reports of strong $\mathrm{PL}$ at $1.87 \mathrm{eV}$. Thus, there is no general explanation for observation of $E_{g} \approx 2.0 \mathrm{eV}$ in some materials, unless one postulates that determinations of low doping and high mobilities in some wide-gap samples were unreliable.

In a second line of attack, Schubina et al. suggested that excess In the MBE growth can produce metallic In clusters leading to a Mie-scattering effects in absorption, which would mask a true energy gap around $1.1 \ldots 1.4 \mathrm{eV}$ (the same authors later appeared to accept the possibility of $E_{g}$ as low as $0.9 \mathrm{eV}$ ). Curiously enough, this "intermediate" gap was observed or deduced in several experiments. The emission near $0.7 \mathrm{eV}$ would then be attributed to transitions at the interface between the InN matrix and the In inclusions. However, the presence of In clusters was ruled out for some MBE-grown materials displaying narrow-gap emission and absorption.

Several reviews of the InN materials properties and energy-gap controversy have appeared, although full understanding remains exlusive at this stage. Since most of the materials with high optical quality display longwave emission, we conjunction with Varshni parameters $\alpha=0.414 \mathrm{meV} \cdot \mathrm{K}^{-1}$ and $\beta=154 \mathrm{~K}$.

Thus, best active layer of emitters for solid state neodymium lasers excitation with $\lambda=1,06 \mu \mathrm{m}$ there are solid state solutions InGaN, which can emitted photons with $\lambda$ less $1,06 \mu \mathrm{m}$.

\section{Experiment and results}

In this work, we used the principle proposed earlier [6] and consisting in reducing the series resistance of nanoregions contact to heterostructures on substrates by formation of surface nanocarbonized structures, such as graphene, fullerenes, nanotubes carbide (Fig. 1).

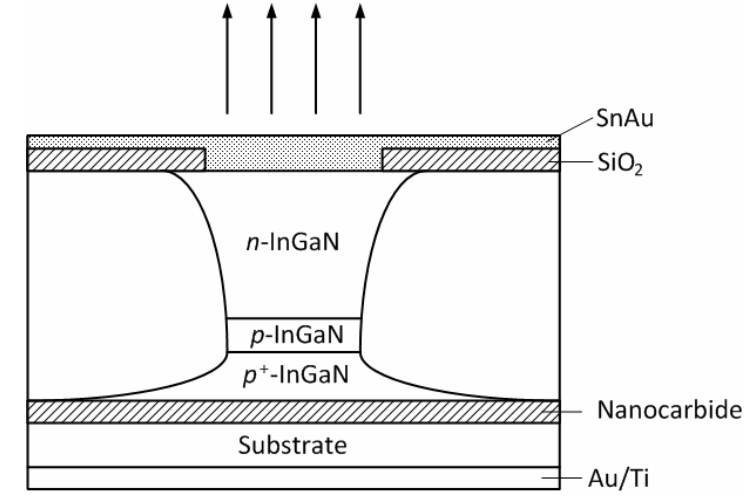

Fig. 1. Structure of a superluminescent laser diode chip with nanocarbide graphene, fullerenes and carbide nanotubes $\mathrm{Al}_{2} \mathrm{O}_{3}$ layer on the substrate of sapphire $\mathrm{Al}_{2} \mathrm{O}_{3}$ or $\mathrm{Si} / \mathrm{SiO}_{2}$.

The electronic and holes conductivity layers creates by internal electric fields from polarization or graded alloys of $\mathrm{A}^{3} \mathrm{~B}^{5}$ or $\mathrm{A}^{2} \mathrm{~B}^{6}$ compounds with variable composition of anion elements $\left(\mathrm{N}, \mathrm{P}, \mathrm{As}, \mathrm{Sb}\right.$ for $\mathrm{B}^{5}$ or $\mathrm{O}, \mathrm{S}, \mathrm{Se}, \mathrm{Te}$ for $\mathrm{B}^{6}$ ) and cation elements $\mathrm{Al}, \mathrm{Ga}$, In for $\mathrm{A}^{3}, \mathrm{Mg} \mathrm{Zn}, \mathrm{Cd}$ for $\mathrm{A}^{2}$ from a high concentration to low one.

The device with the electronic conductivity layer includes quaternary graded $\mathrm{CdZnISe}$ solid state solution, and that with the hole conductivity one is based on quaternary graded $\mathrm{Mg}_{\mathrm{x}} \mathrm{Zn}_{1-\mathrm{x}} \mathrm{O}_{\mathrm{y}} \mathrm{Se}_{1-\mathrm{y}}$ or quaternary $\mathrm{Al}_{\mathrm{x}} \mathrm{Ga}_{1-\mathrm{x}} \mathrm{N}_{\mathrm{y}} \mathrm{As}_{1-\mathrm{y}}$ solid state solution.

One of the important issues of band gap materials and devices is the ohmicity of the contact. In this work, we propose to use narrow graded band gap compositions to reach a low series resistance $\left(\mathrm{ZnSnSb}_{2}, \mathrm{CdSnAs}_{2}\right.$ and $\mathrm{CdSnSb}_{2}$ ). Complex use of $\mathrm{A}_{2} \mathrm{~B}_{4} \mathrm{C}_{2}^{5}$ and III-N heterostructures enabled us to create the highperformance multi-layer structure of LEDs or LDs (Fig. 2).

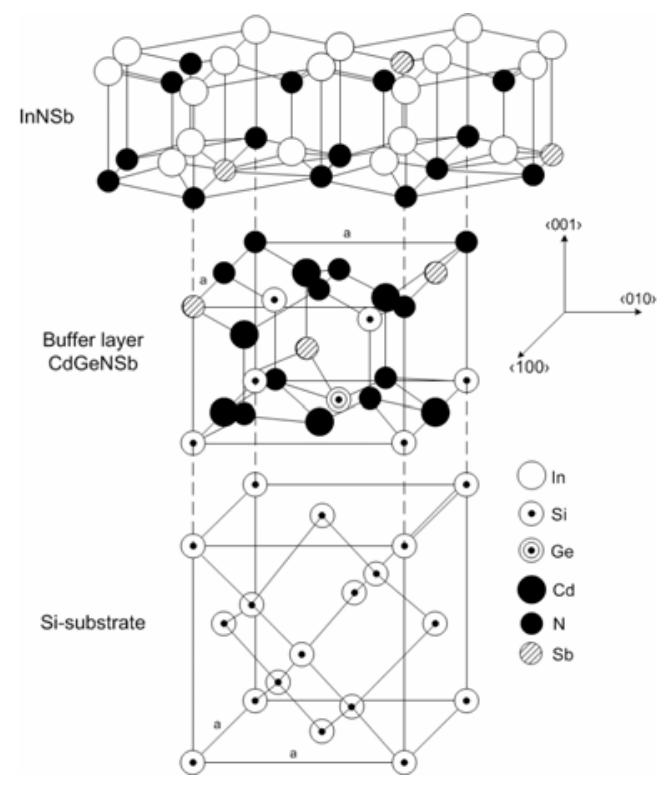

Fig. 2. Structure model of Si/buffer layer CdGeNSb interface. 
The solid state solutions BeMgZnCdSiGeSnN ${ }_{2} \mathrm{P}_{2} \mathrm{As}_{2} \mathrm{Sb}_{2}$ gives the possibility to grow a heterojunction without differences in lattice matching and graded band gap as well as lattice parameters by changing atomic composition between layers $\mathrm{A}_{2} \mathrm{~B}_{4} \mathrm{C}_{2}^{5}$ and with III-N LED structures.

Energy band gap and crystal lattice engineering make it possible to obtain high quality multilayer heterostructures with high light absorption by using the structural buffer layer $\mathrm{A}_{2} \mathrm{~B}_{4} \mathrm{C}_{2}^{5}$ for input of a small part of light into $\mathrm{Si}$ photosensitive structures of microprocessor integrated circuit and effective output of light.

In this case, it is possible to use for these materials double beam refraction and photonic crystal topology. One of the problem in wide-band materials and devices is the ohmic contact. We proposed to use graded narrow band gap compositions $\mathrm{ZnSnSb}, \mathrm{CdSnAs}_{2}$ and $\mathrm{CdSnSb}_{2}$ (Fig. 3) for a low contact resistance. Complex usefulness of $\mathrm{A}_{2} \mathrm{~B}_{4} \mathrm{C}_{2}^{5}$ III-N heterostructures gives the possibility to design multilayer structures.

When creating the integrated LED arrays, we used principles of technological assembly of integrated circuits based on flexible aluminium polyimide carrier. Flexible carriers are used as a system of direct connection to the pads of the crystals and, therefore, the output pins of the hardware, as well as the implementtation of a communication control system with cascade LEDs in solving practical problems [1]. Moreover, the principle of design implementation for enhanced heat removal from the crystals by using reverse mounting. The essence of the above is explained in Fig. 4.

At the first stage of the method of two-sided foil dielectric photolithography, flexible carrier is prepared with the necessary configuration, accounting for geometrical sizes and topology of the chip contact pads as well as board assembly equipment, and in accordance with the scheme of the electrical switching device ready. The flexible carrier, by ultrasonic welding, joins the necessary amount of crystals (1). It should be noted that in this case the crystals may be with contact pads of both gold and aluminum, than is possible to provide a highly reliable monometallic structure by simultaneous selection of metals. Such a module, face down, glued (7) on the heat sink (base case) (6). The heat sink may be made of any material having appropriate thermal characteristics, since the difference in thermal expansion coefficients of the crystal and the heat sink is compensated by elastic adhesive (7). In our case, we chose $1 \ldots 1.5-\mathrm{mm}$ thick aluminium. The assembled structure was covered with a reflector, and lens thereon was formed from organic material. If necessary, to use high power LEDs, crystals of which require to form large pads, the flexible carrier is projected with a ramified system of connecting portions (Fig. 4). Each section is independently joined to the pads of the crystal. The resulting increased area of weld "crystal findings", which improves the heat removal from the chip and also reduces the contact resistance "pad-output".

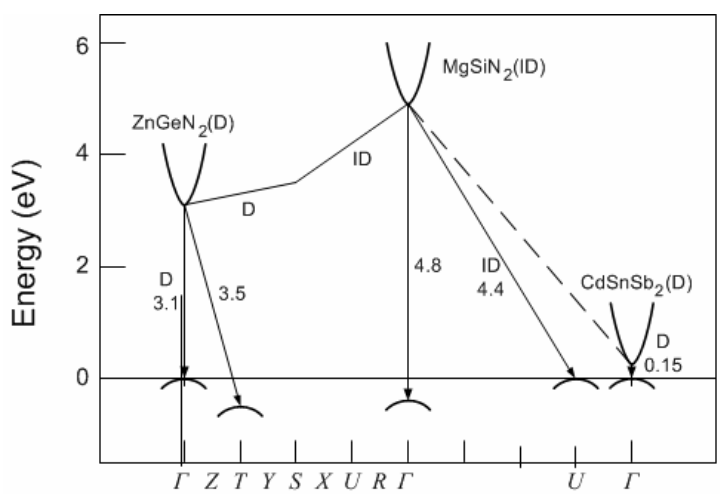

Fig. 3. Brillouin energy zone transformation by changing the composition of $\mathrm{A}_{2} \mathrm{~B}_{4} \mathrm{C}_{2}^{5}$ alloys.

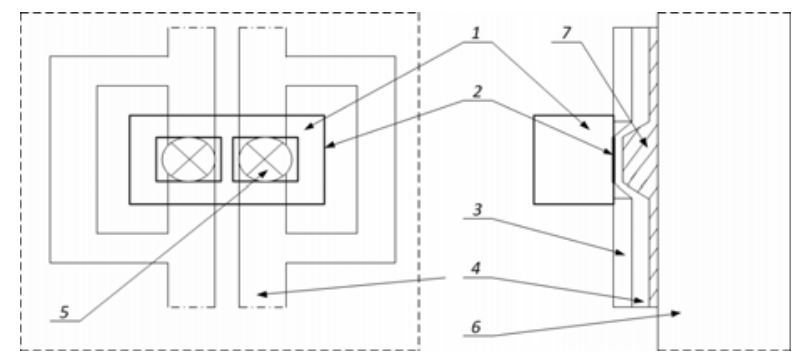

Fig. 4. Fragment of an integrated LED matrix. 1 - LED chip; 2 - pads on the chip; 3 - flexible polyimide carrier; 4 aluminium flexible carrier; 5 - the weld; 6 - heat sink base; 7 glue.

The method for assembling ICs on a flexible carrier may be used in manufacturing the diodes, in which the contact pads are formed on opposite faces of the crystal. In this case, one terminal is welded to the underside of the pad and the other - to the top platform of the crystal.

The curves in Fig. 5 show the lighting characteristics of the proposed design. For comparison, experiments were carried out on the integrated LED matrix with thermally conductive aluminium (a) and pyroceramics basis (b).

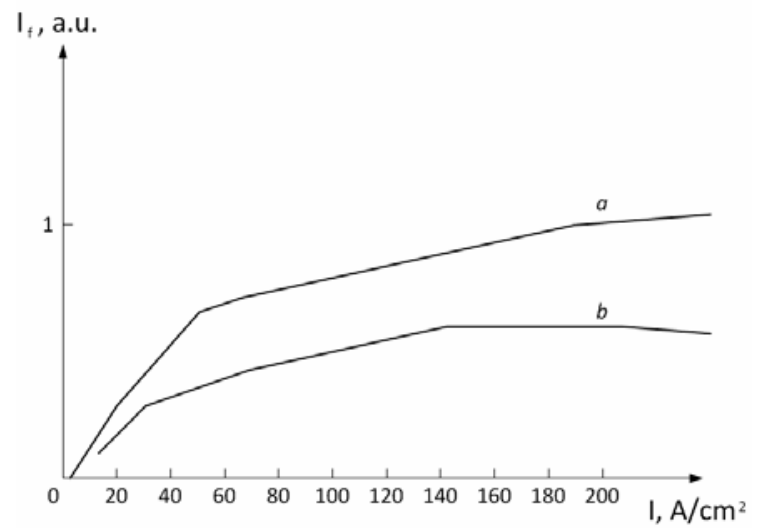

Fig. 5. The dependence of the radiation power of the matrixes based on aluminium (a) and piroceramics (b) in relative units of forward current density. 
Fig. 6 shows the lighting characteristics of matrixes with heat conducting tap at different operating temperatures. When the ambient temperature changes from -40 up to $+70{ }^{\circ} \mathrm{C}$ performances of the flip-chip LED assembly is kept, and the change in the optical power of chips of high-brightness LEDs is a promising technology providing high parameters of radiation in a wide temperature range, high design and technological possibilities of heat dissipation, the light flux controlling. The developed technology allows LED multichip assembly of large format with complex topology and is compatible with transistor technology build integrated devices on a flexible carrier.

It is possible to increase the current through the diode structure without significant reducing the external quantum yield at room and higher temperatures (Fig. 2). The external quantum yield almost linearly decreases with increasing temperature (curve 1) and the output power increases in proportion to the density of current (curve 2). The corresponding parameters shown in Fig. 2 with dashed lines 3 and 4 are reduced due to chip heating by the series resistance of contacts [6]. In structures with nanocarbonized contact layers (curve 2), the increase in efficiencies and power output was approximately 2-fold, which testifies to the effectiveness of reducing the heat on a consistent contact resistance. From comparison of the obtained dependences, the conclusion follows that the gain in efficiency and output power at superluminescent mode enables to reduce the barrier to entry into the laser mode is about 2 times, which improves all operational parameters required to pump neodymium lasers. This opens up the possibility the creation of new generation of neodymium lasers with semiconductor pumping without forced cooling. Under these conditions, the integrated matrix may switch a mode of spontaneous, superluminescent laser radiation control by programming the microprocessor manufactured on a silicon substrate.

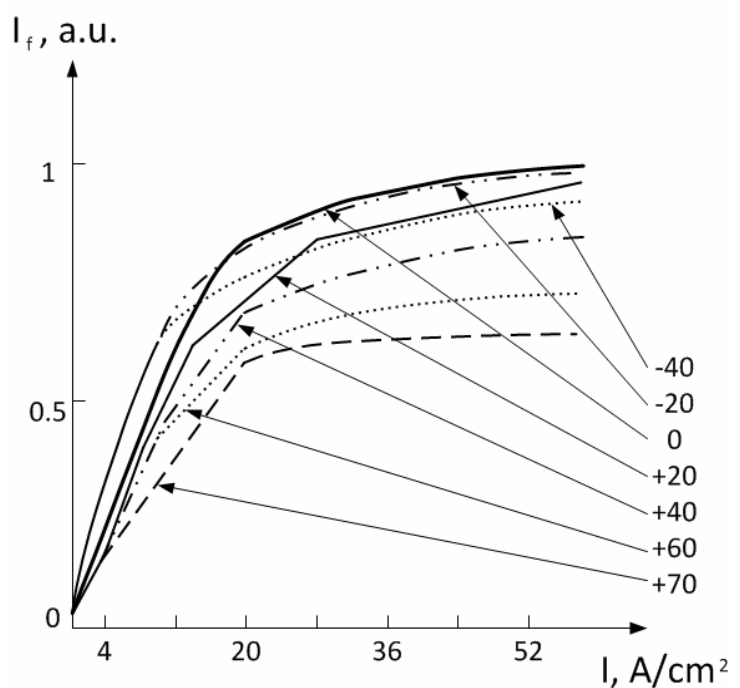

Fig. 6. The dependence of the matrix radiation power density of the direct current at ambient temperatures from -40 up to $+70^{\circ} \mathrm{C}$.

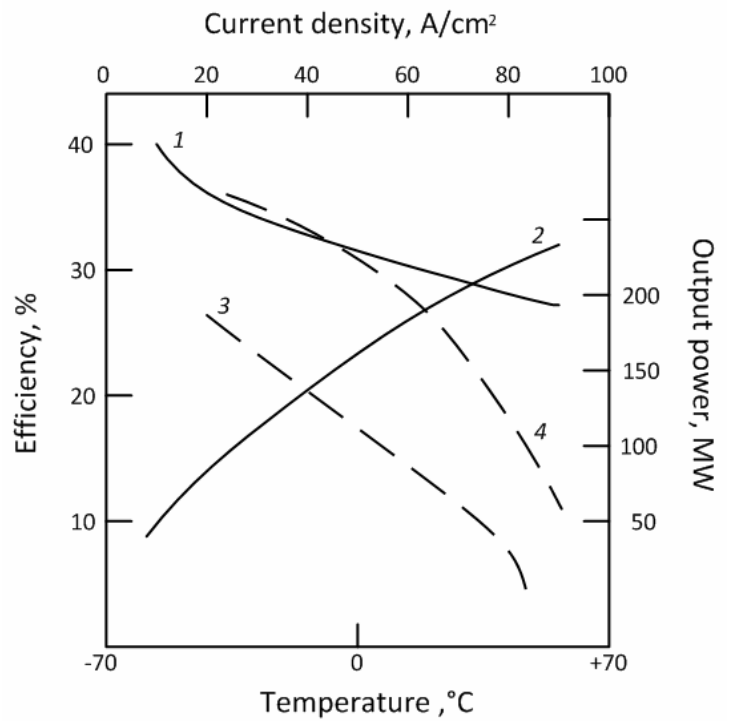

Fig. 7. Temperature and fluorescent (current) dependences of efficiency (\%) and the output power of superluminescent laser heterostructures nanocarbide contact layers $(1,2)$ and without them $(3,4)$.

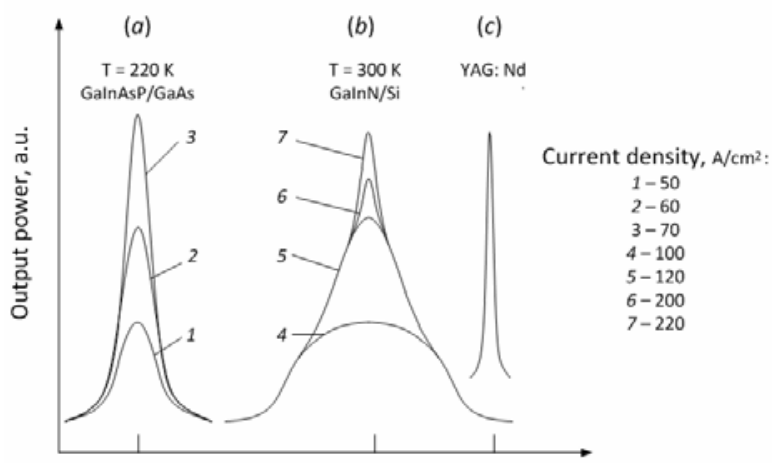

Fig. 8. Spontaneous $(1,4)$, superluminescent $(2,5)$ and the laser $(3,6)$ modes of heterostructure GaInAsP/GaAs $(a)$ with a Peltier-cooled and heterostructures GaInN/Si (b) with nanocarbide epitaxial structures without cooling, and the line of generation inherent to a neodymium laser $(c)$.

Traditional LED heterolaser structures AlGaAs/GaAs have been made on substrates of gallium arsenide, which leads to their emission at wavelengths 820 to $840 \mathrm{~nm}$, far distinguishable from that of the power neodymium laser (Fig. 8a). This results in significant losses of the pumping power. Using heterostructures of III-nitride $\mathrm{AlGaInN/Si}$ allows more accurately select and grow the best chemical composition of the emitting region, which excludes these losses (Fig. 8b). In addition, the dynamics of the crystal lattice of III-nitride less sensitive to changes in temperature $[7,8]$, which enables one to work without additional cooling. Technology integration of LED's matrix on flexible polyimide substrates allows to create any geometry of the luminous flux for spatial optimization of the system at low current densities when pumping neodymium lasers (Fig. 8c). 


\section{Conclusion}

For the first time analytically and experimentally designed hybrid integrated matrixes with $\lambda \approx 0,98 \mu \mathrm{m}$ on the flexible substrates for excitation of neodymium lasers YAG:Nd. It was achieved increasing of efficiency and output power in graded band and carbonized structures with decreasing of heat release by optimization of contact resistance. It was obtained room temperature work of neodymium lasers without forced cooling. The experimental investigation of hybrid packaged LED and LD chips shows the way of monolithic one chip integration of infrared sources on the InGaN structures.

\section{Acknowledgements}

In conclusion, I express my appreciation to Dr. V. Osinsky for formulating the problem, N. Lyakhova and N. Sukhoviy for their assistance in the discussions and in the preparation of heterostructures.

\section{References}

1. V. Osinsky, I. Tuchinsky, V. Zhora, V. Grunianska, N. Liahova, A. Rubanchuk, Flip-chip LED packing technology used flexible carriers on polyimide base // Elektronika i svyaz', temat. vypusk "Problemy elektroniki”, No.2, p. 5-8 (2006), in Russian.
2. V. Osinsky, P. Deminskyi, N. Liahova, A. Motorny, I. Masol, N. Suhoviy, The temperature and concentration properties of $\mathrm{Si} / \mathrm{A}^{\mathrm{III}} \mathrm{B}^{\mathrm{V}} \mathrm{RGB}$ light sources // Optyko-elektronni informatsiynoenergetychni tehnologii, 2(24), p. $50-57$ (2012) in Russian.

3. V. Osinsky, N. Kostiukevich, Integral Optron // Patent of USSR No. 551730, 1973.

4. V. Osinsky, A. Polyakov, G. Gorokh, N. Liahova, S. Pearton, Non-polar $\mathrm{GaN}$ prepared on $\mathrm{Si}$ substrates by hydride vapor phase epitaxy using anodized Al nanomask // Appl. Phys. Lett. 94, 022114 (2009).

5. J. Piprek, Nitride Semiconductor Devices. WileyVCH Verlag GmbH \& Co., 2007.

6. V. Osinsky, M. Stolpovoy, Research of planar contact to the quantum-nanolayers heterostructures n+-GaAs/n-GaAs/n-GaAlAs/n+-GaAs // Optoelektronika i poluprovodnikovaya tehnika, 29, p. 18-24 (1995) in Russian.

7. V. Osinsky, I. Masol, M. Onachenko, A. Sushiy, Decoherence of III-N low-dimensional nanostructures quantum processors // IX Vserossiyskaya konferencia "Gallium, Indium, Aluminium Nitrides and Structures and Devices”, Moscow, June 14-16, 2013, p. 100-101 (in Russian).

8. I. Masol, V. Osinsky, O. Sergeev, Information Nanotechnology. Makros, Kyiv, 2011 (in Russian). 